EUROPEAN JOURNAL OF PURE AND APPLIED MATHEMATICS

Vol. 11, No. 1, 2018, 79-89

ISSN 1307-5543 - www.ejpam.com

Published by New York Business Global

\title{
On ideals and commutativity of prime rings with generalized derivations
}

\author{
M. K. Abu Nawas ${ }^{1, *}$, Radwan M. Al-Omary ${ }^{2}$ \\ 1 Department of Mathematics, Faculty of Science, Northern Border University, Arar, \\ Saudi Arabia \\ 2 Department of Mathematics, Ibb University, Yemen
}

\begin{abstract}
An additive mapping $F: R \rightarrow R$ is called a generalized derivation on $R$ if there exists a derivation $d: R \rightarrow R$ such that $F(x y)=x F(y)+d(x) y$ holds for all $x, y \in R$. It is called a generalized $(\alpha, \beta)$-derivation on $R$ if there exists an $(\alpha, \beta)$-derivation $d: R \rightarrow R$ such that the equation $F(x y)=F(x) \alpha(y)+\beta(x) d(y)$ holds for all $x, y \in R$. In the present paper, we investigate commutativity of a prime ring $R$, which satisfies certain differential identities on the left ideals of $R$. Moreover some results on commutativity of rings with involutions that satisfy certain identities are proved.
\end{abstract}

2010 Mathematics Subject Classifications: 16D90, 16W25, 16N60, 16 U80

Key Words and Phrases: Left ideals, prime rings, centralizing, derivations, generalized derivations, commutativity

\section{Introduction}

Recently, a considerable number of researchers have investigated the ideals in prime rings as well as the commutativity of prime rings that consider derivations and generalized derivations, see for example [2], [3], [5] and [7]. In [4], Ashraf and Khan showed that a *-ideal $U$ is central if the ring $R$ admits a general derivation $F$ associated with a derivation $d$ satisfying specific properties. In [10], El-Soufi and Aboubakr proved that $J \subseteq Z(R)$ under specific properties, where $R$ is a 2 -torsion free prime ring with center $\mathrm{Z}(\mathrm{R})$ admitting a generalized derivation $F$ associated with a derivation $d, J$ is a nonzero Jordan ideal. In addition, Ibraheem in [11] showed that if $f$ is a generalized reverse derivation on $R$ such that $f$ is commuting and centralizing on a right ideal $I$ of $R$, then $R$ is a commutative, where $R$ is a prime ring and $d$ is a reverse derivation on $R$. Moreover, in [1], Abu Nawas and Al-Omary investigated the commutativity of $R$ such that $R$ is a $*$-prime ring admitting

${ }^{*}$ Corresponding author.

Email addresses: m.abunawas.math.nbu@gmail.com (M. Abu Nawas), radwan959@yahoo.com (Radwan Al-Omary) 
generalized $(\alpha, \beta)$ - derivations $F$ and $G$ associated with $(\alpha, \beta)$-derivations $d$ and $g$, respectively, that satisfying certain properties. Let $R$ be an associative ring with center $Z(R)$. For $x, y \in R$ denote the commutator $x y-y x$ by $[x, y]$ and the anti-commutator $x y+y x$ by $x \circ y$. Recall that a ring $R$ is prime if for any $a, b \in R, a R b=\{0\}$ implies that $a=0$ or $b=0$. An additive mapping $d: R \longrightarrow R$ is called a derivation if $d(x y)=d(x) y+x d(y)$ for all $x, y \in R$. In particular, for a fixed $a \in R$, the mapping $I_{a}: R \longrightarrow R$ given by $I_{a}(x)=[x, a]$ is a derivation called an inner derivation. An additive mapping $x \mapsto x^{*}$ on a ring $R$ is called an involution if $\left(x^{*}\right)^{*}=x$ and $(x y)^{*}=y^{*} x^{*}$ for all $x, y \in R$. A ring $R$ equipped with an involution $*$ is said to be a $*$-prime ring if $a R b=a R b^{*}=\{0\}$ implies $a=0$ or $b=0$ for any $a, b \in R$.

An additive function $F: R \longrightarrow R$ is called a generalized inner derivation if $F(x)=a x+x b$ for fixed $a, b \in R$. For such a mapping $F$, it is easy to see that

$$
F(x y)=x F(y)+[a, x] y=x F(y)+I_{a}(x) y \text { for } \text { all } x, y \in R .
$$

This observation leads to the following definition, given in [9]: an additive mapping $F$ : $R \longrightarrow R$ is called a generalized derivation with associated derivation $d$ if

$$
F(x y)=x F(y)+d(x) y \text { for all } x, y \in R .
$$

Familiar examples of generalized derivations are derivations and generalized inner derivations that include left multipliers and right multipliers. Since the sum of two generalized derivations is a generalized derivation, every map of the form $F(x)=x c+d(x)$, where $c$ is a fixed element of $R$ and $d$ is a derivation, is a generalized derivation; and if $R$ has 1 , all generalized derivations have this form. Let $\alpha$ and $\beta$ be endomorphisms of $R$. We shall write for any pair of $x, y \in R,[x, y]_{\alpha, \beta}=x \alpha(y)-\beta(y) x,(x \circ y)_{\alpha, \beta}=x \alpha(y)+\beta(y) x$ An additive map $d: R \longrightarrow R$ is called an $(\alpha, \beta)$-derivation if $d(x y)=d(x) \alpha(y)+\beta(x) d(y)$ for all $x, y \in R$. An additive mapping $F: R \longrightarrow R$ is called a generalized $(\alpha, \beta)$-inner derivation if $F(x)=a \alpha(x)+\beta(x) b$, for some fixed $a, b \in R$ and for all $x \in R$. An additive map $F: R \longrightarrow R$ is called a generalized $(\alpha, \beta)$-derivation associated with an $(\alpha, \beta)$-derivation $d: R \longrightarrow R$ if

$$
F(x y)=F(x) \alpha(y)+\beta(x) d(y) \text { for all } x, y \in R .
$$

Over the last four decade, several authors have proved results on commutativity of prime rings or semiprime rings that admitting automorphisms, derivations or generalized derivations which are centralizing or commuting on appropriate subset of $R$ (see [5], [8], [12] , [14]-[16]).

In this paper, we investigate the commutativity of a prime ring $R$ admitting generalized derivations $F$ and $G$ satisfying any one of the following properties: (i) $F(x) \circ x \in Z(R),(i i)$ $[F(x), F(y)]-F[x, y] \in Z(R),(i i i) F(x) \circ F(y)-F(x \circ y) \in Z(R),(i v) F[x, y]+[F(x), y]-$ $[F(x), F(y)] \in Z(R),(v) F(x \circ y)-[x, y] \in Z(R),(v i)[F(x), F(y)]-x \circ y \in Z(R),(v i i)$ $[F(x), G(y)]-[x, y] \in Z(R),($ viii $)[F(x), x]-[x, G(x)] \in Z(R)$ and $F(x) \circ x-x \circ G(x) \in$ $Z(R)$ for all $x, y$ in some appropriate subset of $R$. Some results on commutativity of rings with involutions that satisfy certain identities are also proved. 


\section{Preliminaries}

We shall use, without explicit mention, the following basic identities that hold for any $x, y, z \in R$ :

$$
\begin{gathered}
{[x y, z]=x[y, z]+[x, z] y ;} \\
{[x, y z]=y[x, z]+[x, y] z ;} \\
x \circ(y z)=(x \circ y) z-y[x, z]=y(x \circ z)+[x, y] z ; \\
(x y) \circ z=x(y \circ z)-[x, z] y=(x \circ z) y+x[y, z] ; \\
{[x y, z]_{\alpha, \beta}=x[y, z]_{\alpha, \beta}+[x, \beta(z)] y=x[y, \alpha(z)]+[x, z]_{\alpha, \beta} y ;} \\
{[x, y z]_{\alpha, \beta}=\beta(y)[x, z]_{\alpha, \beta}+[x, y]_{\alpha, \beta} \alpha(z) ;} \\
(x \circ(y z))_{\alpha, \beta}=(x \circ y)_{\alpha, \beta} \alpha(z)-\beta(y)[x, z]_{\alpha, \beta}=\beta(y)(x \circ z)_{\alpha, \beta}+[x, y]_{\alpha, \beta} \alpha(z) ; \\
((x y) \circ z)_{\alpha, \beta}=x(y \circ z)_{\alpha, \beta}-[x, \beta(z)] y=(x \circ z)_{\alpha, \beta} y+x[y, \alpha(z)] .
\end{gathered}
$$

The following results are also going to be used:

Remark 2.1. In a prime ring, the centralizer of any nonzero one-sided ideal is equal to the center of $R$; in particular, if $R$ has nonzero central ideal, $R$ must be commutative.

Remark 2.2. Let $R$ be a prime ring. For a nonzero element $a \in Z(R)$, if $a b \in Z(R)$, then $b \in Z(R)$.

We begin our discussion with the following results.

Lemma 2.1. Let $R$ be a prime ring. If $d: R \longrightarrow R$ is a derivation on $R$, then for any $0 \neq z \in Z(R), d(z) \in Z(R)$.

Proof. We have $0 \neq z \in Z(R)$, that is $[z, r]=0$ for all $r \in R$ and hence $d[z, r]=0$, $d(z r-r z)=0$, i.e $d(z) r+z(d(r)-d(r) z-r d(z)=0$, that is $[d(z), r]+[z, d(r)]=0$, since $z \in Z(R)$ so we get $[d(z), r]=0$ for all $r \in R$ which yields that $d(z) \in Z(R)$.

Lemma 2.2. [[6], Theorem 2] Let $R$ be a prime ring and $I$ a nonzero left ideal of $R$ such that $I \cap Z(R) \neq 0$. If $R$ admits a generalized derivation $F$ with associated derivation $d$ such that $F$ is centralizing on $I$, then $R$ is commutative.

Lemma 2.3. [[7], Theorem 4] Let $R$ be a prime ring and $I$ a nonzero left ideal. If $R$ admits a nonzero derivation $d$ such that $[d(x), x] \in Z(R)$ for all $x \in I$, then $R$ is commutative.

Lemma 2.4. [[13], Lemma 2.5] If a prime ring $R$ contains a nonzero commutative right ideal, then $R$ is commutative.

Lemma 2.5. Let $R$ be a prime ring and $I$ be a nonzero left ideal of $R$ such that

(a) $[x, y] \in Z(R)$ for all $x, y \in I$, or

(b) $x \circ y \in Z(R)$ for all $x, y \in I$. 
Then $R$ is commutative.

Proof. (a) We have $[x, y] \in Z(R)$ for all $x, y \in I$. This implies that $[r,[x, y]]=0$ for all $r \in R$. Replace $y$ by $y x$ in the above relation, to get $[r,[x, y x]]=[r,[x, y] x]=$ $[x, y][r, x]+[r,[x, y]] x=0$, now use the relation $[r,[x, y]]=0$, to get $[x, y][r, x]=0$ for all $x, y \in I, r \in R$. Again, replace $r$ by $r y$ to get $[x, y] R(-[x, y])=\{0\}$ for all $x, y \in I$ and primeness of $R$ yields that $[x, y]=0$ for all $x, y \in I$ and hence by Lemma 2.4, $R$ is commutative.

(b) If $x \circ y \in Z(R)$ for all $x, y \in I$, then $[x \circ y, r]=0$ for all $r \in R$. Replacing $y$ by $y x$ we find that $(x \circ y)[x, r]=0$. For any $s \in R$, replace $r$ by $s r$ to get $(x \circ y) R[x, r]=\{0\}$. Thus, for each $x \in I$ either $x \circ y=0$ or $[x, r]=0$. Let $A=\{x \in I \mid x \circ y=0$ for all $y \in I\}$, $B=\{x \in I \mid[x, r]=0$ for all $r \in R\}$. Then $A$ and $B$ are additive subgroups of $I$ whose union is $I$. But a group cannot be the union of two proper subgroups and hence either $x \circ y=0$ for all $x, y \in I$ or $[x, r]=0$ for all $x \in I$ and $r \in R$. If $x \circ y=0$, then replace $y$ by $r y$ we obtain $[x, r] y=0$ for all $x, y \in I$ and $r \in R$, that is $[x, r] I=\{0\}$. Since $I \neq 0$, we get $[x, r]=0$ for all $x \in I$ and $r \in R$ and both the cases we find that $I$ is central and hence by Remark $2.1, R$ is commutative.

\section{Main Results}

Theorem 3.1. Let $R$ be a prime ring and $I$ a nonzero left ideal of $R$. Suppose that $R$ admits a generalized derivation $F$ with associated derivation $d$ of $R$ such that $d(Z(R)) \neq 0$. Further, if $R$ satisfies the condition $F(x) \circ x \in Z(R)$ for all $x \in I$, then $R$ is commutative. get

Proof. By hypothesis we have $F(x) \circ x \in Z(R)$ for all $x \in I$. Replace $x$ by $x+y$, to

$$
F(x) \circ y+F(y) \circ x \in Z(R) \text { for all } x, y \in I .
$$

Since $d(Z(R)) \neq 0$, then there exists $z \in Z(R)$ such that $d(z) \neq 0$. Replace $y$ by $z y$ in (1) and using (1), we get

$$
[F(x), z] y-[z, x] F(y)+d(z)(y \circ x)-[d(z), x] y \in Z(R) .
$$

Now by Lemma 2.1, $d(z) \in Z(R)$ and therefore we find that $d(z)(y \circ x) \in Z(R)$. Since $R$ is prime and $d(z) \neq 0$, it follows from Remark 2.2 that $y \circ x \in Z(R)$ for all $x, y \in I$ and hence by Lemma $2.5(\mathrm{~b}), R$ is a commutative.

Theorem 3.2. Let $R$ be a prime ring and $I$ a nonzero left ideal of $R$ such that $I \cap Z(R) \neq 0$. Suppose that $R$ admits a generalized derivation $F$ with associated derivation d such that $d(Z(R)) \neq 0$. Further, if $R$ satisfies any one of the following conditions:

(i) $[F(x), F(y)]-F[x, y] \in Z(R)$ for all $x, y \in I$, or 
(ii) $F(x) \circ F(y)-F(x \circ y) \in Z(R)$ for all $x, y \in I$,

then $R$ is commutative.

Proof. (i) For all $x, y \in I$, we have

$$
[F(x), F(y)]-F[x, y] \in Z(R) .
$$

Since $d(Z(R)) \neq 0$, then there exists $z \in Z(R)$ such that $d(z) \neq 0$. Replacing $y$ by $z y$ in (2) and using (2), we get $[F(x), z] F(y)+[F(x), d(z)] y+d(z)([F(x), y]-[x, y]) \in Z(R)$ for all $x, y \in I$. Since $d(z) \in Z(R)$ by Lemma 2.1, and therefore $d(z)([F(x), y]-[x, y]) \in Z(R)$. Since $d(z) \neq 0$ and $R$ is prime, it follows from Remark 2.2 that $[F(x), y]-[x, y] \in Z(R)$ for all $x, y \in I$. Now, replace $y$ by $d(z) x$ in the above relation and use it, to get $d(z)[F(x), x] \in Z(R)$. Again using the same arguments as used above we find that $[F(x), x] \in Z(R)$ for all $x \in I$. Thus, by Lemma 2.2 we get $R$ is commutative.

(ii) For all $x, y \in I$, we have

$$
F(x) \circ F(y)-F(x \circ y) \in Z(R) .
$$

Since $d(Z(R)) \neq 0$, then there exists $z \in Z(R)$ such that $d(z) \neq 0$. Replacing $y$ by $z y$ in (3) and using (3), we get $[F(x), z] F(y)+d(z)(F(x) \circ y-x \circ y)+[F(x), d(z)] y \in Z(R)$. Now by Lemma $2.1 d(z) \in Z(R)$ and therefore $d(z)(F(x) \circ y-x \circ y) \in Z(R)$. Since $d(z) \neq 0$ and $R$ is prim, hence from Remark 2.2 we find that $F(x) \circ y-x \circ y \in Z(R)$ for all $x, y \in I$. Again replace $x$ by $z x$ in the last expression and use it, to get $d(z)(x \circ y)-[d(z), y] x \in Z(R)$. Now by Lemma $2.1 d(z) \in Z(R)$ and therefore $d(z)(x \circ y) \in Z(R)$ and hence by Remark 2.2 , we obtain $x \circ y \in Z(R)$ for all $x, y \in I$. Thus, by Lemma $2.5(b)$, we conclude that $R$ is commutative.

Theorem 3.3. Let $R$ be a prime ring and $I$ a nonzero left ideal of $R$ such that $I \cap Z(R) \neq 0$. Suppose that $R$ admits a generalized derivation $F$ with associated derivation d such that $d(Z(R)) \neq 0$. Further, if $R$ satisfies the condition:

$$
F[x, y]+[F(x), y]-[F(x), F(y)] \in Z(R) \text { for all } x, y \in I,
$$

then $R$ is commutative.

Proof. For all $x, y \in I$, we have

$$
F[x, y]+[F(x), y]-[F(x), F(y)] \in Z(R) .
$$

Since $d(Z(R)) \neq 0$, then there exists $z \in Z(R)$ such that $d(z) \neq 0$. Replacing $y$ by $z y$ in (4) and using (4), we get $d(z)([x, y]-[F(x), y])-[F(x), d(z)] y \in Z(R)$. Since by Lemma 2.1 $d(z) \in Z(R)$ and hence by Remark 2.2 we obtain, $[F(x), y]+[x, y] \in Z(R)$ for all $x, y \in I$. Again replace $y$ by $d(z) x$ in the last relation and use it, to get $d(z)[F(x), x] \in Z(R)$ for all $x \in I$, again using the same arguments as above we find that $[F(x), x] \in Z(R)$ for all $x \in I$ and hence by Lemma $2.2, R$ is commutative. 
Theorem 3.4. Let $R$ be a prime ring and $I$ a nonzero ideal of $R$. Suppose that $R$ admits a generalized derivation $F$ with associated derivation d such that $d(Z(R)) \neq 0$. Further, if $R$ satisfies any one of the following conditions:

(i) $F(x \circ y)-[x, y] \in Z(R)$ for all $x, y \in I$, or

(ii) $F(x \circ y)+[x, y] \in Z(R)$ for all $x, y \in I$,

then $R$ is commutative.

Proof. (i) By hypothesis we have $F(x \circ y)-[x, y] \in Z(R)$ for all $x, y \in I$. If $F=0$, then $[x, y] \in Z(R)$ for all $x, y \in I$, and hence by Lemma $2.5(a)$, we get the required result. Therefore we shall assume that $F \neq 0$, then we have for any $x, y \in I$

$$
F(x \circ y)-[x, y] \in Z(R) .
$$

Since $d(Z(R)) \neq 0$, then there exists $z \in Z(R)$ such that $d(z) \neq 0$. Replace $y$ by $z y$ in $(5)$ to get

$$
z F(x \circ y)+d(z)(x \circ y)-z[x, y] \in Z(R), \text { for all } x, y \in I,
$$

and hence by (5), we find that $d(z)(x \circ y) \in Z(R)$ for all $x, y \in I$. Thus, Lemma 2.1 and Remark 2.2 gives that $x \circ y \in Z(R)$ and hence by Lemma 2.5(b) we get the required result.

(ii) Using the same trick as used in (i), result follows.

Theorem 3.5. Let $R$ be a prime ring and $I$ a nonzero left ideal of $R$ such that $I \cap Z(R) \neq 0$. Suppose that $R$ admits a generalized derivation $F$ with associated derivation $d$ such that $d(Z(R)) \neq 0$. Further, if $R$ satisfies any one of the following conditions:

(i) $[F(x), F(y)]-x \circ y \in Z(R)$, for all $x, y \in I$, or

(ii) $[F(x), F(y)]+x \circ y \in Z(R)$, for all $x, y \in I$,

then $R$ is commutative.

Proof. (i) By hypothesis we have $[F(x), F(y)]-x \circ y \in Z(R)$ for all $x, y \in I$. If $F=0$, then $x \circ y \in Z(R)$ for all $x, y \in I$, and hence we get the required result by Lemma 2.5(b). Therefore we shall assume that $F \neq 0$, then for any $x, y \in I$ we have

$$
[F(x), F(y)]-x \circ y \in Z(R) .
$$

Since $d(Z(R)) \neq 0$, then there exists $z \in Z(R)$ such that $d(z) \neq 0$. Replacing $y$ by $z y$ in (6) and using (6), we get $d(z)[F(x), y]+[F(x), d(z)] y \in Z(R)$. Now, since $z \in Z(R)$ then by Lemma 2.1 we have $d(z)[F(x), y] \in Z(R)$ and hence by Remark 2.2, we find that $[F(x), y] \in Z(R)$ for all $x, y \in I$. In particular $[F(x), x] \in Z(R)$ for all $x \in I$ and hence by Lemma $2.2, R$ is commutative.

(ii) Using similar arguments as (i) it follows. 
Theorem 3.6. Let $R$ be a prime ring and $I$ a nonzero left ideal of $R$ such that $I \cap Z(R) \neq 0$. Suppose that $R$ admits a generalized derivations $F$ and $G$ with associated derivations $d$ and $g$ respectively, such that $g(Z(R)) \neq 0$. Further, if $R$ satisfies any one of the following conditions:

(i) $[F(x), G(y)]-[x, y] \in Z(R)$, for all $x, y \in I$, or

(ii) $[F(x), G(y)]+[x, y] \in Z(R)$, for all $x, y \in I$,

then $R$ is commutative.

Proof. Given that $F$ and $G$ are generalized derivations of $R$ such that $[F(x), G(y)]-[x, y] \in$ $Z(R)$ for all $x, y \in I$. If $F=0$ (or $G=0$ ), then $[x, y] \in Z(R)$ for all $x, y \in I$, and hence by Lemma $2.5(a), R$ is a commutative.

Therefore, we shall assume that $F \neq 0$ (and $G \neq 0$ ). For any $x, y \in I$ we have

$$
[F(x), G(y)]-[x, y] \in Z(R) .
$$

Since $g(Z(R)) \neq 0$, then there exists $z \in Z(R)$ such that $g(z) \neq 0$. Replacing $y$ by $z y$ in (7) and using (7), we get $g(z)[F(x), y]+[F(x), g(z)] y \in Z(R)$ for all $x, y \in I$, since by Lemma 2.1, $g(z) \in Z(R)$ so we find that $g(z)[F(x), y] \in Z(R)$. Thus, by Remark 2.2, we find that $[F(x), y] \in Z(R)$ for all $x, y \in I$. In particular $[F(x), x] \in Z(R)$ for all $x \in I$. Hence, $R$ is commutative by Lemma 2.2 .

(ii) Using the same technique as above we get the required result.

Theorem 3.7. Let $R$ be a prime ring and $I$ a nonzero left ideal of $R$ such that $I \cap Z(R) \neq 0$. Suppose that $R$ admits a generalized derivations $F$ and $G$ with associated derivations $d$ and $g$ respectively, such that $\{z \in Z(R) \mid d(z)=g(z) \neq 0\} \neq \phi$. Further, if $R$ satisfies any one of the following conditions:

(i) $[F(x), x]-[x, G(x)] \in Z(R)$, for all $x \in I$, or

(ii) $[F(x), x]+[x, G(x)] \in Z(R)$, for all $x \in I$,

then $R$ is commutative.

Proof. (i) It is given that $F$ and $G$ are generalized derivations of $R$ such that $[F(x), x]-$ $[x, G(x)] \in Z(R)$ for all $x \in I$. If $G=0$ then $[F(x), x] \in Z(R)$ for all $x \in I$, (or if $F=0$, then $-[x, G(x)] \in Z(R)$ ) and hence in both the cases by Lemma 2.2, we get the required result.

Henceforth, we shall assume that $F \neq 0$ (and $G \neq 0$ ). For any $x \in I$, we have $[F(x), x]-$ $[x, G(x)] \in Z(R)$. Linearizing the above expression, we get

$$
[F(x), y]+[F(y), x]-[x, G(y)]-[y, G(x)] \in Z(R) .
$$


Since $\{z \in Z(R) \mid d(z)=g(z) \neq 0\} \neq \phi$. Replacing $y$ by $z y$ in (8) and using (8), we find that $d(z)[y, x]+[d(z), x] y-g(z)[x, y]-[x, g(z)] y \in Z(R)$ for all $x, y \in I$. Since $z \in Z(R)$ and hence by Lemma $2.1, d(z) \in Z(R)$ and $g(z) \in Z(R)$ and therefore $(d(z)+g(z)) \in Z(R)$. Thus, we find that $(d(z)+g(z))[y, x] \in Z(R)$ and hence by Remark 2.2, we get $[y, x] \in Z(R)$ for all $x, y \in I$ and hence by Lemma $2.5(a)$, we get the required result.

(ii) Using similar arguments as above it follows.

Theorem 3.8. Let $R$ be a prime ring and $I$ a nonzero ideal of $R$. Suppose that $R$ admits a generalized derivations $F$ and $G$ with associated derivations $d$ and $g$ respectively, such that $\{z \in Z(R) \mid d(z)=g(z) \neq 0\} \neq \phi$. Further, if $R$ satisfies any one of the following conditions:

(i) $F(x) \circ x-x \circ G(x) \in Z(R)$, for all $x \in I$, or

(ii) $F(x) \circ x+x \circ G(x) \in Z(R)$, for all $x \in I$,

then $R$ is commutative.

Proof. ( $i$ ) It is given that $F$ and $G$ are generalized derivations of $R$ such that $F(x) \circ x-x \circ$ $G(x) \in Z(R)$ for all $x \in I$. If $F=0$, then $F(x) \circ x \in Z(R)$ for all $x \in I$, (or if $G=0$, then $-(x \circ G(x)) \in Z(R)$ and hence in both the cases by Theorem 3.1 we get the required result.

Henceforth, we shall assume that $G \neq 0$ (and $F \neq 0$ ). For any $x \in I$, we have $F(x) \circ x-$ $x \circ G(x) \in Z(R)$. Linearizing the last expression, to get

$$
F(x) \circ y+F(y) \circ x-x \circ G(y)-y \circ G(x) \in Z(R) .
$$

Since $\{z \in Z(R) \mid d(z)=g(z) \neq 0\} \neq \phi$. Replace $y$ by $z y$ in (9) and use (9), to get $d(z)(y \circ x)-[d(z), x] y-g(z)(x \circ y)-[x, g(z)] y \in Z(R)$ for all $x, y \in I$. Hence, by Lemma 2.1 we find that $d(z) \in Z(R)$ and $g(z) \in Z(R)$ and therefore $d(z)-g(z) \in Z(R)$. Thus, we obtain $(d(z)-g(z))(y \circ x) \in Z(R)$ and by Remark 2.2 it follows that $y \circ x \in Z(R)$ for all $x, y \in I$ and hence $R$ is commutative by Lemma $2.5(b)$.

(ii) Using similar arguments as above it follows.

In the next theorem, we consider two identities involving generalized $(\alpha, \beta)$-derivation $F$ associated with $(\alpha, \beta)$-derivation $d$, such that $R$ is a prime ring with involution $*$, and we show that $R$ is commutative.

Theorem 3.9. Let $R$ be a 2-torsion free $*$-prime ring and $\alpha, \beta$ be automorphisms on $R$. If $R$ admits a generalized $(\alpha, \beta)$-derivation $F$ associated with a nonzero $(\alpha, \beta)$-derivation $d$ such that either 
(i) $F[x, y]-[F(x), y]_{\alpha, \beta}=[d(y), x]_{\alpha, \beta}$ for all $x, y \in R$, or

(ii) $F[x, y]-(F(x) \circ y)_{\alpha, \beta}=[d(y), x]_{\alpha, \beta}$ for all $x, y \in R$,

then $R$ is commutative.

Proof. ( $i$ ) If $F=0$, then we have $[d(y), x]_{\alpha, \beta}=0$ for all $x, y \in R$. Replacing $y$ by $y x$ in the last expression gives $\beta(y)[d(x), x]_{\alpha, \beta}+[\beta(y), \beta(x)] d(x)=0$. Again replacing $y$ by $z y$ gives $[\beta(z), \beta(x)] \beta(y) d(x)=0$ for all $x, y, z \in R$. But as $\beta$ is an automorphism on $R$, we have

$$
[\beta(z), \beta(x)] R d(x)=0 \text { for all } x, z \in R .
$$

If $x \in S_{*}(R) \cap R$, then $\beta([z, x]) R d(x)=\beta([z, x])^{*} R d(x)$. Thus, for some $x \in S_{*}(R) \cap R$, the $*$-primeness of $R$ yields either $\beta([z, x])=0$ or $d(x)=0$. But for any $x \in R$, $x-x^{*} \in S_{*}(R) \cap R$. Thus, for some $x \in R$, either $\left[z, x-x^{*}\right]=0$ or $d\left(x-x^{*}\right)=0$. If $\left[z, x-x^{*}\right]=0$, then equation (10) follows that $\beta([z, x]) R d(x)=0=\beta([z, x])^{*} R d(x)$. Hence the $*$-primeness of $R$ yields either $\beta([z, x])=0$ or $d(x)=0$. If $d\left(x-x^{*}\right)=0$ then $d(x)=(d(x))^{*}$ for all $x \in R$. Consequently, for all $x, z \in R$, either $\beta([z, x])=0$ or $d(x)=0$. Let $A=\{x \in R \mid d(x)=0\}$ and $B=\{x \in R \mid[z, x]=0\}$. Then $A$ and $B$ are both additive subgroups of $R$ whose union is $R$. Using Brauer's trick we have either $A=R$ or $B=R$. If $A=R$ then $d(x)=0$ for all $x \in R$, a contradiction. If $B=R$, then $[z, x]=0$ for all $x, z \in R$ and hence $R$ is commutative.

Therefore, we shall assume that $F \neq 0$. So, for any $x, y \in R$, we have

$$
F[x, y]-[F(x), y]_{\alpha, \beta}=[d(y), x]_{\alpha, \beta} .
$$

Replacing $y$ by $y x$ in (11) gives

$$
\beta(y)[x, x]_{\alpha, \beta}+2 \beta([x, y]) d(x)=\beta(y)[d(x), x]_{\alpha, \beta} \text {, for all } x, y \in R .
$$

Again we replace $y$ by $w y$ in (12) to get $2 \beta([x, w]) \beta(y) d(x)=0$ for all $x, y, w \in R$. Since $R$ is a 2 -torsion free and $\beta$ is an automorphism, we get $\beta([x, w]) R d(x)=\{0\}$, for all $x, w \in R$. Therefore, proceeding in the same way as that after (10), gives the required result.

(ii) If $F=0$, then we have $[d(y), x]_{\alpha, \beta}=0$ for all $x, y \in R$. Applying the same techniques as that used above to prove $(i)$ yields the required result. Henceforth, we shall assume that $F \neq 0$. So, for all $x, y \in R$, we have

$$
F[x, y]-(F(x) \circ y)_{\alpha, \beta}=[d(y), x]_{\alpha, \beta} .
$$

Replacing $y$ by $y x$ in (13) gives

$$
\beta(y)[x, x]_{\alpha, \beta}+2 \beta([x, y]) d(x)=\beta(y)[d(x), x]_{\alpha, \beta} .
$$

Again, we replace $y$ by $w y$ in $(14)$ to get $2 \beta([x, w]) \beta(y) d(x)=0$ for all $x, y \in R$. Since $R$ is a 2 -torsion free and $\beta$ is an automorphism on $R$, we get

$$
\beta([x, w]) R d(x)=\{0\}, \text { for all } x, w \in R .
$$

Now, using similar techniques as that after equation (10), we get the required result. 


\section{Acknowledgement}

This paper was financially supported by The Deanship of Scientific Research, Northern Border University, under the project no. 435-062-7. The authors would like to thank The Deanship of Scientific Research for their financial support. The authors also would like to thank Professor Nadeem ur Rehman for many useful comments.

\section{References}

[1] M. K. Abu Nawas and R. M. Al-Omary. On Commutativity of *-Prime Rings with Generalized ( $\alpha, \beta)$-Derivations. Ultra Engineer, 3(1):1-5, (2015).

[2] R. M. Al-Omary, and N. Rehman. Lie ideals and Centralizing mappings with generalized derivations. Journal of Scientific Research and Reports, 11(3):1-8, (2016).

[3] M. Ashraf, A. Ali And S. Ali. Some Commutativity theorems for rings with generalized derivations. Southeast Asian Bull. Math., 32(2):415-421, (2007).

[4] M. Ashraf and A. Khan. Commutativity *-prime rings with generalized derivations. Rend. Sem. Mat. Univ. Padova, 125:71-79, (2011).

[5] M. Ashraf and N. Rehman. On Commutativity of rings with derivations. Results Math., 42:3-8, (2002).

[6] A. Asif and T. Shah. Centralizing and commuting Generalized Derivation On Prime Rings. Matema., 60:1-2, (2008).

[7] H. E. Bell and W.S. Martindale. Centralizing and commuting Generalized Derivation On Prime Rings. Centralizing mapping of semiprime rings. Canda. Math. Bull., 30:92-101, (1987).

[8] H. E. Bell and N. Rehman. Generalized derivations with commutativity and anti-commutativity conditions. Math. J. Okayama Univ., 49:139-147, (2007).

[9] M. Bresar. Centralizing mapping and derivations in prime rings. J.Algebra., 156:385-394, (1993).

[10] M. El-Soufi And A. Aboubakr. Generalized derivations on Jordan ideals in prime rings. Turk. J. Math., 38:233-239, (2014).

[11] A. Ibraheem. Right Ideals and Generalized Reverse Derivations on Prime Rings. American Journal of Computational and Applied Mathematics, 6(4):162-164, (2016).

[12] H. Marubayashi, M. Ashraf, N. Rehman and S. Ali. On generalized $(\alpha, \beta)$ derivations in prime rings. Algebra Colloquim, 17(1):865-874, (2010). 
[13] N. Rehman, S. Mallikarjuna and V. V. Kumar. On commutativity of rings with generalized derivations. Math. J. Okayama Univ., 44:43-49, (2002).

[14] N. Rehman, R. M. Al-Omary and C. Haetinger. On Lie structrue of prime ring with generalized $(\alpha, \beta)$-derivations. Bol. Soc. Paranaense de Mat., 27(2):43$52,(2009)$.

[15] N. Rehman and R. M. Al-Omary. On Commutativity of 2-torsion free *-prime Rings with Generalized Derivations. Mathematica, 53 (76):171-180, (2011).

[16] N. Rehman, R. M. Al-Omary and H. Shuliang. Lie ideals and generalized $(\alpha, \beta)$-derivations of $*$-prime Rings. Africa Mathematica, 24 (4):503510, (2013). 